\title{
Radiomic Feature Analysis for Distinguishing Solitary Pulmonary Capillary Hemangioma From Lepidic-Predominant Lung Adenocarcinoma in Ground Glass Lung Nodules
}

\author{
Mong-Wei Lin \\ National Taiwan University Hospital \\ Hao-Jen Wang \\ National Taiwan University \\ Yi-Chang Chen \\ National Taiwan University Hospital \\ Li-Wei Chen \\ National Taiwan University \\ Min-Shu Hsieh \\ National Taiwan University Hospital \\ Shun-Mao Yang \\ National Taiwan University \\ Ho-Feng Chen \\ National Taiwan University \\ Chuan-Wei Wang \\ National Taiwan University \\ Jin-Shing Chen \\ National Taiwan University Hospital \\ Yeun-Chung Chang \\ National Taiwan University Hospital \\ Chun-Ming Chen ( $\nabla$ chung@ntu.edu.tw ) \\ National Taiwan University
}

\section{Research Article}

Keywords: computed tomography, ground-glass nodule, lung adenocarcinoma, lung cancer surgery, solitary pulmonary capillary hemangioma

Posted Date: December 15th, 2020 
DOl: https://doi.org/10.21203/rs.3.rs-125479/v1

License: (c) (1) This work is licensed under a Creative Commons Attribution 4.0 International License. Read Full License 


\section{Abstract}

Solitary pulmonary capillary hemangioma (SPCH) is a benign lung tumor that presents as ground-glass nodules (GGN) on computed tomography (CT) images, mimicking lepidic-predominant adenocarcinoma (LPA). This study aimed to establish a discriminant model using a radiomic feature analysis to distinguish SPCH from LPA in lung GGNs. This study included 13 and 49 patients who underwent complete resection for lung SPCH and LPA, respectively. An SPCH/LPA classification model was proposed based on a two-level decision tree and 26 radiomic features extracted from each segmented lesion, including 5 and 21 features from the histogram and co-occurrence matrix, respectively. The twolevel decision tree was constructed based on the training data with a support vector machine (SVM) as the classifier in each tree node. For comparison, a baseline model was built with the same 26 features using an SVM as the classifier. Both models were assessed by the leave-one-out cross-validation method. The area under the receiver operating characteristic curve, accuracy, sensitivity, and specificity of the proposed SPCH/LPA model were $0.954,91.9 \%, 92.3 \%$, and $91.8 \%$. The proposed SPCH/LPA model significantly outperformed the baseline model $(p<0.05)$. Our results may help surgeons to preoperatively discriminate SPCH from LPA, thus avoiding unnecessary surgery for benign tumors.

\section{Introduction}

Solitary pulmonary capillary hemangioma (SPCH) is a primary benign lung tumor that exhibits proliferation of capillaries in the alveolar septa [1]. This uncommon disease was first described in a report of eight autopsies in 2000 as "pulmonary capillary hemangiomatosis-like foci" [2], and the term SPCH was first used in a report of two surgically resected patients in 2006 [3]. Only 17 cases of surgically resected SPCH have been reported in the English literature before 2018 [1, 3-11]. Clinically, SPCH usually presents as a ground-glass nodule (GGN) on chest computed tomography (CT) images, which is similar to the presentation of adenocarcinoma in situ, minimally invasive adenocarcinoma, and lepidicpredominant adenocarcinoma (LPA) of the lung [1, 3-13].

Recently, the Dutch-Belgian lung cancer screening trial NELSON showed that lung cancer mortality is significantly lower among high-risk persons who underwent CT screening than among those who did not undergo screening [14]. CT has been accepted as an effective lung cancer screening tool for high-risk patients $[14,15]$. In about $20 \%$ of the screened population, the screening detected indeterminate lung nodules; these patients may need further management [16]. In those, it is not uncommon that surgical resection reveals such lung nodules to be benign lung tumors, including SPCH. Recently, more cases of surgically resected SPCH have been reported [12, 13]. In 2018, Hsieh et al. described a series of 16 surgically resected SPCH patients [12]. All SPCHs were incidentally detected by CT screening, and all 16 SPCH lesions were initially unrecognized or misdiagnosed by general pathologists. Their report showed that SPCH may be underrecognized by radiologists in CT scans due to its similarity to early lung cancer and may also be underrecognized by general pathologists. 
To assist in the identification of SPCH in thoracic CT images, the present study aimed to extract radiomic features that could preoperatively distinguish SPCH from LPA in lung GGNs. Although numerous radiomic features have been developed for the differential diagnosis of lung nodules [17], the differentiation of SPCH from LPA remains a difficult task because both types of lesions have very similar GGN-like appearances. Moreover, the limited number of cases reported for SPCH makes the training dataset intrinsically imbalanced. To overcome these difficulties, here, a radiomic analysis rooted in a divide-and-conquer paradigm is proposed to construct a two-level radiomic feature set as the basis of separating SPCH from LPA.

\section{Results}

\section{Demographic and clinicopathological characteristics}

The characteristics of the 13 SPCH patients and 49 LPA patients investigated in our study are summarized in Table 1. In the SPCH group, a predominance of females (53.8\%) and nonsmokers (92.3\%) was observed. The mean tumor diameter was $10.1 \mathrm{~mm}$. The CT findings showed pure GGN, part-solid GGN, and pure solid in $4(30.8 \%), 8(61.5 \%)$, and 1 (7.7\%) patients, respectively. Similar to the SPCH group, female (71.4\%) and nonsmoker (89.8\%) predominance was also observed in the LPA group. In this group, most tumors were classified as pure GGN (71.4\%) in CT images. The pathological features revealed noninvasive characteristics in most cases of the LPA group. The lepidic percentages were higher than $70 \%$ in all cases of the LPA group. Besides, neither micropapillary nor solid subtypes were noted in the enrolled patients. 
Table 1

Clinicopathological features of study cohort

\section{$\mathrm{SPCH}$}

Number of patients

Age (years)

Female

Nonsmoker

Lung cancer family history

Abnormal CEA

Tumor location

LUL

LLL

RUL

RML

RLL

Tumor diameter in CT image $(\mathrm{mm})$

CT findings

Pure GGN

Part solid GGN

Solid

Tumor differentiation

Well

Moderate

Visceral pleural invasion

Lymphovascular invasion

Lymph node metastasis
13

$54.5 \pm 12.6(38-77)$

7 (53.8)

$12(92.3)$

$4(30.8)$

$1(7.7)$

1 (7.7)

$14(28.6)$

4 (30.8)

8 (16.3)

$4(30.8)$

20 (40.8)

1 (7.7)

$3(6.1)$

$3(23.1)$

$10.1 \pm 3.8(6-19)$

4 (8.2)

$11.2 \pm 5.9(5-28.9)$

35 (71.4)

$14(28.6)$

8 (61.5)

$0(0)$

1 (7.7)

43 (87.8)

$6(12.2)$

$0(0)$

$0(0)$

Data are presented as mean \pm SD or number (\%).

Abbreviations: CEA, carcinoembryonic antigen; CT, computed tomography; GGN, ground-glass nodule; LLL, left lower lobe; LPA, lepidic-predominant adenocarcinoma; LUL, left upper lobe; RLL, right lower lobe; RML, right middle lobe; RUL, right upper lobe; $\mathrm{SPCH}$, solitary pulmonary capillary hemangioma. 


\begin{tabular}{|ll|}
\hline \multicolumn{1}{|c|}{ SPCH } & LPA \\
\hline Lepidic percentage & $8(16.3)$ \\
\hline $70-80 \%$ & $2(4.1)$ \\
\hline $80-90 \%$ & $22(44.9)$ \\
\hline $90-100 \%$ & $16(32.7)$ \\
\hline Pure lepidic & \\
\hline Data are presented as mean \pm SD or number (\%). & \\
\hline $\begin{array}{l}\text { Abbreviations: CEA, carcinoembryonic antigen; CT, computed tomography; GGN, ground-glass nodule; } \\
\text { LLL, left lower lobe; LPA, lepidic-predominant adenocarcinoma; LUL, left upper lobe; RLL, right lower } \\
\text { lobe; RML, right middle lobe; RUL, right upper lobe; SPCH, solitary pulmonary capillary hemangioma. }\end{array}$ \\
\hline Analysis Of radiomic features
\end{tabular}

Radiomic feature analysis involved histogram features and 3D spatial texture features. The mean values of seven features, including kurtosis $(p=0.026)$, uniformity $(p<0.001)$, autocorrelation $(p<0.001)$, correlation $(p<0.001)$, sum of squares: variance $(p<0.001)$, sum average $(p<0.001)$, and sum variance $(p<0.001)$, were significantly different between SPCH and LPA. The detailed statistical analyses of the histogram features and the 3D spatial texture features are listed in Table 2. 
Table 2

Histogram and 3D spatial texture feature analyses for patients with solitary pulmonary capillary hemangioma and lepidic predominant adenocarcinoma

\begin{tabular}{|c|c|c|c|}
\hline & SPCH & LPA & P-value \\
\hline Numbers of Patients & 13 & 49 & \\
\hline \multicolumn{4}{|l|}{ Histogram features } \\
\hline Skewness & $1.413 \pm 0.773$ & $1.227 \pm 0.511$ & 0.302 \\
\hline Kurtosis & $4.888 \pm 3.70$ & $3.406 \pm 1.415$ & 0.026 \\
\hline 75th percentile & $87.75 \pm 33.976$ & $80.505 \pm 30.563$ & 0.461 \\
\hline 97.5th percentile & $128.55 \pm 54.810$ & $100.971 \pm 35.913$ & 0.106 \\
\hline Uniformity & $0.0173 \pm 0.005$ & $0.029 \pm 0.011$ & $<0.001$ \\
\hline \multicolumn{4}{|c|}{ Tumor region feature analysis by GLCM } \\
\hline Autocorrelation & $54.450 \pm 43.098$ & $215.698 \pm 186.057$ & $<0.001$ \\
\hline Contrast & $5.179 \pm 4.420$ & $3.667 \pm 3.529$ & 0.198 \\
\hline Correlation & $0.501 \pm 0.091$ & $0.636 \pm 0.121$ & $<0.001$ \\
\hline Cluster Prominence & $973.146 \pm 982.489$ & $2051.079 \pm 5967.805$ & 0.521 \\
\hline Cluster shade & $-8.520 \pm 60.754$ & $62.069 \pm 168.781$ & 0.145 \\
\hline Dissimilarity & $1.546 \pm 0.742$ & $1.255 \pm 0.719$ & 0.202 \\
\hline Energy & $0.049 \pm 0.026$ & $0.066 \pm 0.062$ & 0.141 \\
\hline Entropy & $3.512 \pm 0.579$ & $3.470 \pm 0.955$ & 0.844 \\
\hline Inverse Difference & $0.554 \pm 0.099$ & $0.603 \pm 0.136$ & 0.230 \\
\hline Inverse Difference Moment & $0.505 \pm 0.121$ & $0.563 \pm 0.165$ & 0.242 \\
\hline Maximum probability & $0.114 \pm 0.044$ & $0.127 \pm 0.109$ & 0.518 \\
\hline Sum of squares: Variance & $57.70 \pm 45.249$ & $216.875 \pm 186.892$ & $<0.001$ \\
\hline Sum average & $13.245 \pm 5.961$ & $25.005 \pm 15.119$ & $<0.001$ \\
\hline Sum variance & $160.969 \pm 142.297$ & $735.150 \pm 651.642$ & $<0.001$ \\
\hline Sum entropy & $2.481 \pm 0.347$ & $2.492 \pm 0.526$ & 0.930 \\
\hline
\end{tabular}

Values were presented as mean \pm standard deviation.

Abbreviations: LPA, lepidic predominant adenocarcinoma; $\mathrm{SPCH}$, solitary pulmonary capillary hemangioma. 


\begin{tabular}{|lclc|}
\hline & SPCH & LPA & P-value \\
\hline Difference variance & $5.179 \pm 4.420$ & $3.667 \pm 3.529$ & 0.198 \\
\hline Difference entropy & $1.487 \pm 0.341$ & $1.306 \pm 0.415$ & 0.153 \\
\hline Information measure of correlation 1 & $-0.170 \pm 0.052$ & $-0.197 \pm 0.075$ & 0.234 \\
\hline Information measure of correlation 2 & $0.663 \pm 0.099$ & $0.685 \pm 0.106$ & 0.507 \\
\hline Inverse difference normalized & $0.994 \pm 0.003$ & $0.995 \pm 0.003$ & 0.202 \\
\hline Inverse difference moment normalized & $0.999 \pm 0.00007$ & $0.999 \pm 0.00005$ & 0.202 \\
\hline Values were presented as mean \pm standard deviation. & & \\
\hline $\begin{array}{l}\text { Abbreviations: LPA, lepidic predominant adenocarcinoma; SPCH, solitary pulmonary capillary } \\
\text { hemangioma. }\end{array}$ & & \\
\hline
\end{tabular}

\section{Performance of the SPCH/LPA classification model}

For the root node of the two-level decision tree, two principal components corresponding to the two largest eigenvalues were extracted from the training data in each fold of the leave-one-out crossvalidation process. The results of this process showed that the two principal components, on average, explained $99.88 \% \pm 0.61 \%$ of the total variance of all training data in the 26 -dimensional feature space in every fold. For the leaf node, only four texture features were selected, namely correlation, inverse difference, uniformity, and information measure of correlation 2, which accounted for $61,37,14$, and 12 times of feature selection, respectively, amounting to all 124 times of feature selection in the 62 folds of training processes.

The receiver operating characteristic (ROC) curve of the proposed SPCH/LPA classification model is illustrated in Fig. 1, which was plotted based on the SPCH probability of each test datum, i.e., $p=p 1 \times p 2$. The area under the curve (AUC) of the ROC curve was 0.954 . The classification accuracy, sensitivity, and specificity of the proposed SPCH/LPA classification model were $91.9 \%, 92.3 \%$, and $91.8 \%$, respectively, when a decision threshold of 0.5 was applied to the SPCH probability $p$.

\section{Performance of the baseline model}

Three to six features were selected by the sequential forward feature selection (SFFS) algorithm for each of the 62 folds in the leave-one-out cross-validation process as listed in Supplementary Table 1, adding up to 285 times of feature selection in total. The total number of selections for each feature is summarized in Supplementary Table 2. The six most selected features were uniformity, correlation, dissimilarity, autocorrelation, energy, and inverse difference, which were selected 62, 62, 35, 20, 18, and 17 times, respectively, representing $75.1 \%$ of feature selection in the 62 folds of training processes.

Figure 1 also displays the ROC curve of the baseline model with an AUC of 0.805 . The classification accuracy, sensitivity, and specificity of this baseline model were $85.5 \%, 61.5 \%$, and $91.8 \%$, respectively, for 


\section{Discussion}

Advances in CT imaging and the availability of CT screening programs for lung cancer detection in asymptomatic high-risk patients have increased the detection rate for small pulmonary nodules $[15,16]$. There are several guidelines and recommendations for the management and follow-up of incidentally encountered lung nodules detected by CT in adult patients [26, 27]. According to these recommendations, some indeterminate lung nodules are indicated for surgical resection. However, the final diagnosis of these indeterminate lung nodules includes not only malignancies but also benign lung tumors [16]. SPCH has been an uncommon benign lung tumor, with only 17 reported cases of surgically resected SPCH before 2018 [1, 3-11]. However, 25 more cases have been reported in the last two years [12, 13]. The increasing incidence of this benign tumor raised the importance of preoperative diagnosis. However, SPCH is characterized by GGNs in CT images and mimics LPA radiographically [1, 3-13]. Even experienced thoracic surgeons and radiologists may regard SPCH as early primary lung cancer. Therefore, it is important to differentiate SPCH from LPA by radiomic feature analysis. Our results showed that SPCH could be differentiated from LPA on CT images by a radiomic texture feature-based classification model.

Radiomic texture features have been widely used in tissue characterization for discriminating different types of lesions in thoracic CT images, such as automatic lung nodule detection [28-36], differential diagnosis of benign and malignant lung nodules [15, 16], and differentiation of lung cancer subtypes [17]. Several texture features have been suggested for automated detection of pulmonary nodules in CT images, including the mean, skewness, and kurtosis values of intensity histograms [28-33], local binary patterns $[9,14,34,35]$, and gray-level co-occurrence matrix (GLCM)-based features [28-30, 36]. While radiomic texture features were shown to be effective in distinguishing various types of lung nodules, discrimination between SPCH and LPA was intrinsically difficult due to their common GGN-like appearance. Moreover, this task was challenging because the limited number of available SPCH cases evidently made the study population highly imbalanced.

The difficulty caused by the common GGN-like appearance could be partially observed in the similar texture features of the enrolled SPCH and LPA cases. As listed in Table 2, only 7 out of 26 radiomic features showed significant differences between the mean values of SPCH and LPA for the 62 lesions examined in this study. What made the differentiation between SPCH and LPA even more difficult was that the coefficients of variation (CVs), i.e., (standard deviation / mean) · 100, of these 26 features were mostly very high (>30), suggesting a high heterogeneity among each of the SPCH and LPA cohorts. As a result, the baseline model could only achieve an AUC of 0.805 with a combination of three to six features.

Despite the texture similarity between SPCH and LPA and the intra-cohort heterogeneity revealed in Table 2, different texture characteristics between SPCH and LPA might be qualitatively inferred from four radiomic features, including kurtosis, uniformity, sum average, and correlation. These four features, 
referred to as characteristic appearance features hereinafter, had relatively low $\mathrm{CV}$ s and significant differences between SPCH and LPA individually. Firstly, a lower kurtosis implied a lighter tail and a higher uniformity meaning a more homogeneous gray-level distribution; this suggested that on average, an LPA was relatively more homogeneous than an $\mathrm{SPCH}$. Secondly, a higher sum average and a higher correlation indicated a larger mean of sum and a higher linear correlation for the horizontally adjacent pairs of voxel intensities, respectively. Combining the implications of these four features suggested that on average, the intensities of horizontally adjacent voxel pairs of an LPA tended to be higher and more similar to each other than those of an SPCH. These observations were also partly supported by the mean values of some other features, such as lower contrast, lower dissimilarity, and higher inverse difference, in the LPA group though their textures were not significantly different. Noted that the characteristic appearance features were identified by inference without actually constructing a discrimination model, which should not be confused with the radiomic features selected by the SFFS algorithm.

To cope with the problems of appearance similarity, cohort heterogeneity, and imbalanced samples, a divide-and-conquer radiomic analysis approach was proposed in this study. A two-level SPCH/LPA decision tree was proposed with the essential idea of partitioning the LPA samples into two subsets. One subset comprised lesions with high confidence of being an LPA, whereas the other was expected to contain LPA lesions with higher discriminability from SPCH lesions for the subset was more homogeneous than its parent sample set. This idea was realized at the root node of the employed twolevel decision tree. Specifically, it was accomplished by using the first two principal components of the training data in the 26-dimensional feature space and optimizing an support vector machine (SVM) that maximized the positive predictive value subject to the constraint of $100 \%$ sensitivity. A lesion that was not picked out at the root node, i.e., $p 1=1$, was forwarded to the second level of the decision tree to determine the probability of being an SPCH lesion.

To avoid overfitting of the classification model, only two features were selected in the leaf node based on the training data in each fold of the leave-one-out cross-validation process, following the suggestion given by Jain et al. [24]. Noticeably, only four texture features had been selected in the 62 folds; the three most-selected features were correlation, inverse difference, and uniformity, which amounted to $90.3 \%$ of feature selections. Interestingly, these three features corresponded to three of the four characteristic appearance features which characterized the intensity similarity of horizontally adjacent voxel pairs. More precisely, the intensities of horizontally adjacent voxel pairs of an LPA were inclined to be more similar to each other than those of an SPCH.

The divide-and-conquer radiomic analysis embedded in the two-level SPCH/LPA decision tree demonstrated greatly enhanced discernability of SPCHs from LPAs in comparison to the baseline classification model. The baseline model represented a generic machine learning model using a single set of radiomic features, which was the same set of texture features as used in the proposed two-level SPCH/LPA classification model. The respective AUC and accuracy values were significantly improved from 0.805 and $85.5 \%$ in the baseline model to 0.954 and $91.9 \%$ in the SPCH/LPA classification model. 
This study had several limitations. The first limitation was that tumor heterogeneity exists in lung adenocarcinoma. We tried to include LPA cases with the lepidic subtype of more than $70 \%$ to eliminate histological heterogeneities among analyzed tumors in the LPA group and to extract representative radiomic information of LPA lesions. Second, relatively small numbers of SPCH and LPA cases were enrolled in this study, restricting the ability to account for tumor heterogeneity and texture variation caused by differences in CT scanner models. Because an SPCH diagnosis is uncommon, the number of patients with SPCH will remain limited until more cases are reported. Although the number of enrolled LPA cases might be increased in various ways, this approach would be constrained by the limited number of patients with SPCH due to the persistent data imbalance problem. However, the patient cohort in this study is the largest SPCH cohort in the currently existing literature, and our results are valuable to thoracic surgeons and radiologists worldwide. The accountability limitation for texture variation might have been partially weakened by the multiple varieties of CT scanner models employed in this study, which collated data from three makers and ten models. Lastly, this was a retrospective study and did not exactly represent the real-world setting. The result of this study could only be applied to distinguish between SPCH and LPA, but not to differentiate SPCH from other subtypes of lung adenocarcinoma. Further validation with a larger cohort in a multicenter study is necessary.

In conclusion, our study showed that SPCH could be accurately differentiated from lung cancer on CT images by a radiomic texture feature-based discriminant model. Our results may help surgeons to preoperatively discriminate between patients with SPCH and LPA, thus avoiding unnecessary surgery for benign lung tumors.

\section{Materials And Methods}

\section{Study population and CT acquisition}

Twenty-nine consecutive SPCH cases with tumor size $>5 \mathrm{~mm}$ were considered in this study. These SPCH cases underwent surgical tumor resection by a single surgical team, using the same clinical protocols and perioperative orders, at the National Taiwan University Hospital between January 2013 and December 2017. Out of these 29 cases, 9 cases with other lung nodules in the same lobe were excluded from this study. Another 7 cases without preoperative thin-cut CT images were also excluded. Finally, 13 cases were enrolled in the SPCH group for further analysis (Figure 2). For the patient selection in the LPA group, we retrospectively evaluated 3327 consecutive patients who underwent thoracoscopic surgery for lung cancer by the same surgical team at our institute between January 2013 and December 2018. The inclusion criteria in the LPA group were as follows: 1) diagnosed as lung adenocarcinoma with pathologically confirmed near-pure ( $\geq 70 \%$ ) of lepidic-predominant histological subtypes and 2 ) existence of preoperative thin-cut CT images. Finally, 49 cases were enrolled in the LPA group for further analysis (Figure 2). All pathologic slides of the enrolled patients were reviewed according to the 2015 World Health Organization criteria [18]. The characteristics of the $13 \mathrm{SPCH}$ patients and 49 LPA patients investigated in our study are summarized in Table 1. The SPCH diagnosis and the percentage of the lepidic subtype were confirmed microscopically by an experienced thoracic pathologist (MSH). The Research Ethics 
Committee at the National Taiwan University Hospital reviewed and approved this study (project approval no. 202003074RIND; approval date, April 14, 2020) and waived informed consent for this retrospective study.

\section{Image acquisition}

Chest CT images were obtained with a 16-, 64-, 128-, or 256-detector row CT scanner from the following manufacturers: GE (LightSpeed 16, LightSpeed VCT, Revolution CT, and Revolution RVO), Siemens (Emotion 16, Sensation 64, and SOMATON Definition AS+), Philips (iCT 256, and Ingenuity CT), and Canon (Aquilion ONE) Medical Systems. The CT image parameters were as follows: detector collimation, 0.5-0.625 mm; pitch, $0.813-1.2$; gantry speed, 0.35 or 0.5 s per rotation; $120 \mathrm{kVp} ; 41-330 \mathrm{~mA}$; slice thickness, $1.0-1.25 \mathrm{~mm}$; and matrix, $512 \times 512$.

\section{SPCH/LPA classification model}

The SPCH/LPA classification model was based on a divide-and-conquer radiomic analysis. The kernel idea was to untangle the intervened radiomic distribution of SPCH and LPA by two different sets of radiomic features. The first set partitioned the SPCH/LPA dataset into two subsets, including one with high confidence of being LPA and the other a mixture of SPCH and LPA to be further classified using the second set of radiomic features. The rationale behind this idea was to decompose the LPA samples into two subgroups, each of which was expected to have a higher homogeneity than its parent group. It reduced the originally imbalanced and intertwining classification problem into a relatively balanced problem with a more homogeneous subset of LPA, opening up an opportunity for better discrimination between SPCH and LPA.

The schema of this study is depicted in Figure 3. The SPCH- or LPA-containing volumes of interest were first extracted from 3D thoracic CT images followed by segmentation processes demarcating the lesion boundaries. Texture features were extracted from histograms and the GLCM [19] of the lesions with a divide-and-conquer paradigm. The performance of the proposed SPCH/LPA classification model was assessed using a leave-one-out cross-validation method.

\section{Tumor segmentation}

Segmentation was a key step in the process of extracting radiographic features for the features were extracted from the lesion volumes defined by the derived lesion boundaries. However, the segmentation procedure was a challenging task for SPCH and LPA due to the weak lesion boundaries formed by the GGN-like appearance, as well as the complex structural compositions of blood vessels, bronchial tubes, pleural indentations, among others. To better describe the lesion boundaries of SPCH and LPA, a semiautomatic segmentation algorithm based on the hybrid level-set algorithm proposed by Zhang et al. [20] was developed in the present study. The key ideas were to find a lesion boundary maximizing overall edge gradient strengths while ensuring regional uniformity within the boundary. To further account for the complex compositions of SPCH and LPA, especially, along the lesion boundaries, the computer-generated 
lesion boundaries were examined and, if necessary, modified manually by two chest radiologists (YC Chen and YC Chang) to reach consensus segmentation results.

\section{Feature extraction}

To characterize SPCH and LPA, two types of radiomic features were extracted from the segmented lesions, namely the histogram features and the 3D spatial texture features. The histogram features included skewness, kurtosis, $75^{\text {th }}$ percentile, $97.5^{\text {th }}$ percentile, and uniformity [21]. The 3D spatial texture features were composed of 21 features derived from the GLCM of each lesion as listed in Table 2. The histogram features characterized the gray-level distribution of a lesion, whereas the 3D spatial texture features described the spatial distribution of the gray levels within a lesion. More precisely, the $21 \mathrm{GLCM}$ based texture features modeled the gray-level co-occurrence characteristics of all horizontally adjacent voxels in a lesion.

\section{Feature selection and classification model building}

Based on the key idea of a divide-and-conquer radiomic analysis, the SPCH/LPA classification model, comprising a two-level decision tree with two SVMs, was employed to differentiate SPCH from LPA. The first level of the decision tree, i.e., the root node of the decision tree, was composed of an SVM followed by a step function, $u(P s 1-0.5)$, where $P s 1$ is the probability of a nodule being an SPCH estimated by the SVM with $u(x)=1$ for $x \geqq 0$ and $u(x)=0$ for $x<0$. If we denote the probability output of a nodule at the root node of the decision tree by $P 1$, then $P 1=u(P S 1-0.5)$. The SVM of the root node was constructed based on the training data, the features of which comprised the first two principal components of the training data points in the feature space. The feature space was formed by the 26 features of all training data, including 5 histogram-based and 21 GLCM-based features. The first two principal components were the eigenvectors corresponding to the two largest eigenvalues derived via principal component analysis [22]. The SVM of the root node maximized the positive predictive value subject to the constraint of $100 \%$ sensitivity. A true positive referred to the case that an SPCH was correctly classified as such.

Because the SVM of the root node was trained to have a sensitivity of $100 \%$, the second level of the decision tree consisted of only one leaf node to further classify the positive outcomes of the root node into SPCH or LPA. The classifier of the leaf node was also an SVM but without being connected to a step function. The SVM of the leaf node was built using the 26 texture features. To avoid overfitting the classification model, a subset of features was selected to best differentiate SPCH from LPA by a SFFS algorithm [23] using the training dataset. While there is no unique rule in determining the maximum number of features for $n$ training data, Jain et al. [24] suggested that the number of features used for a two-class classification problem should be less than $n / 10$. Considering the reduced number of training data at the leaf node, the number of features to be selected to train the SVM was set to 2 in this study. The SVM of the leaf node yielded the probability of a nodule being an $\mathrm{SPCH}$, denoted by $P 2$. As a result, the probability of a test datum being classified as an $\mathrm{SPCH}$ would be $P=P 1 \times P 2$. It should be noted that for a test datum with $P 1=0$, its probability of being an $\mathrm{SPCH}$ was set to 0 , i.e., $P=0$. 


\section{Performance assessment}

To evaluate the performance of the proposed classification model, a leave-one-out cross-validation approach [25] was used in the present study to estimate the model's ability in predicting the new data that were not involved in the model construction. With a total of 62 cases, the leave-one-out cross-validation carried out 62 folds of cross-validation in a way that each case took turns to serve as the test data and the remaining 61 cases were treated as the training data. The training data were used not only to derive the two principal components for the root node and select the features for the leaf node but also to construct the two SVMs of the two-level decision tree.

To demonstrate the advantage of the divide-and-conquer radiomic analysis, a baseline classification model was implemented with the aim to separate SPCH from LPA using a single set of radiomic features. The baseline model used the SVM as the classifier with the same set of 26 texture features as the SPCH/LPA classification model. The performance of the baseline model was also evaluated by the leaveone-out cross-validation method. The SFFS algorithm was used to select the features in each fold using the training data. Following the rule suggested by Jain et al. [24], the number of features selected in each fold was no more than 6.

\section{Statistical analysis}

To investigate the differentiation capability of each radiomic feature, the independent two-sample $t$-test was conducted for each of the 26 features. Levene's test was performed prior to the $t$-test to assess the homogeneity of variance of each radiomic feature with the null hypothesis of equal population variances. The significance levels of the independent two-sample $t$-test and Levene's test were both set to 0.05 . If the $p$-value of Levene's test was less than the significance level, the null hypothesis was rejected and both groups, i.e., SPCH and LPA, were considered to have unequal variances for the tested radiomic feature. Otherwise, the group variances of SPCH and LPA were considered as equal.

ROC curve analyses were carried out to assess the leave-one-out cross-validation performances of the proposed SPCH/LPA classification model and the baseline model using the probability of being an SPCH, i.e., $p$.

\section{Declarations}

\section{AUTHOR CONTRIBUTIONS STATEMENT}

Study conceptualization: M-W Lin, C-M Chen; Data curation: M-W Lin, H-J Wang, Y-C Chen, S-M Yang, M-S Hsieh; Investigations and analyses: M-W Lin, H-J Wang, Y-C Chen, M-S Hsieh, H-F Chen, C-W Wang, C-M Chen; Visualization and interpretation of the findings: H-J Wang, Y-C Chen, M-S Hsieh, H-F Chen, C-W Wang; Validation of the results: M-W Lin, H-J Wang, Y-C Chang, C-M Chen; Writing the original draft of the manuscript: M-W Lin, H-J Wang, Y-C Chen, S-M Yang, H-F Chen, C-W Wang; Reviewing and editing the manuscript: L-W Chen, M-S Hsieh, J-S Chen, Y-C Chang, C-M Chen; Funding acquisition, project 
administration, and project supervision: M-W Lin, J-S Chen, Y-C Chang, C-M Chen. All authors have approved the submitted version of the manuscript and have agreed both to be personally accountable for the author's own contributions and to ensure that questions related to the accuracy or integrity of any part of the work, even ones in which the author was not personally involved, are appropriately investigated, resolved, and the resolution documented in the literature.

\section{ADDITIONAL INFORMATION}

Competing interests: The authors declare no competing interests.

Funding: This study was supported by the Ministry of Science and Technology, Taiwan (MOST 107-2221E-002-080-MY3) and National Taiwan University Hospital, Taipei, Taiwan (NTUH109-S4659, MS419).

\section{References}

1. Hashimoto H, Kurata A, Fujiwara M et al. Solitary Pulmonary Capillary Hemangioma of Adult Cases: Clinicopathologic Characteristics as an Unrecognized Entity. Am J Surg Pathol 40:1380-1389 (2016).

2. Havlik DM, Massie LW, Williams WL, Crooks LA. Pulmonary capillary hemangiomatosis-like foci. An autopsy study of 8 cases. Am J Clin Pathol 113:655-662 (2000).

3. Fugo K, Matsuno Y, Okamoto K et al. Solitary capillary hemangioma of the lung: report of 2 resected cases detected by high-resolution CT. Am J Surg Pathol 30:750-753 (2006).

4. Kadowaki T, Yano S, Kobayashi K, Araki K, Tokushima T, Yamadori I. Pulmonary capillary hemangiomatosis-like foci detected by high resolution computed tomography. Intern Med 49:175178 (2010).

5. Saito T, Maniwa T, Kaneda $\mathrm{H}$ et al. Coexistence of catamenial pneumothorax and catamenial hemoptysis in a patient with pulmonary hemangiomatosis-like foci: a case report. $\mathrm{J}$ Thorac Cardiovasc Surg 139:e14-e16 (2010).

6. Matsushita M, Kawakami S, Matsushita T et al. Changes in CT density of solitary capillary hemangioma of the lung upon varying patient position. Jpn J Radiol 30:772-776 (2012).

7. Lee HW, Ahn S, Shim YM, Choi YS, Lee KS, Han J. Solitary capillary hemangioma of the lung: a report of two cases. J. Lung Cancer 11:102-104 (2012).

8. Isaka T, Yokose $\mathrm{T}$, Ito $\mathrm{H}$ et al. Case of solitary pulmonary capillary hemangioma: pathological features based on frozen section analysis. Pathol Int 63:615-618 (2013).

9. Sakaguchi Y, Isowa N, Tokuyasu H, Miura H. A resected case of solitary pulmonary capillary hemangioma showing pure ground glass opacity. Ann Thorac Cardiovasc Surg 20 Suppl:578-581 (2014).

10. Hashimoto $\mathrm{H}$, Yanagiya $\mathrm{M}$, Suzuki $\mathrm{Y}$ et al. A case of solitary pulmonary capillary hemangioma indicating true gross appearance. Pathol Int 67:322-323 (2017). 
11. Zhu Y, Qu N, Sun L, Meng X, Li X, Zhang Y. Solitary pulmonary capillary hemangioma presents as ground glass opacity on computed tomography indicating adenocarcinoma in situ/atypical adenomatous hyperplasia: A case report. Biomed Rep 7:515-519 (2017).

12. Hsieh MS, Lee YH, Lin MW, Chen JS. Solitary pulmonary capillary hemangioma: An under-recognized pulmonary lesion mimicking early lung cancer on computed tomography images. Lung Cancer 124:227-232 (2018).

13. Zhao J, Shao J, Zhu L et al. Solitary pulmonary capillary hemangioma: Clinicopathologic and radiologic characteristics of nine surgically resected cases. Pathol Res Pract 214:1885-1891 (2018).

14. de Koning HJ, van der Aalst CM, de Jong PA et al. Reduced Lung-Cancer Mortality with Volume CT Screening in a Randomized Trial. N Engl J Med 382:503-513 (2020).

15. National Lung Screening Trial Research Team, Aberle DR, Berg CD et al. The National Lung Screening Trial: overview and study design. Radiology 258:243-253 (2011).

16. Lin MW, Tseng YH, Lee YF et al. Computed tomography-guided patent blue vital dye localization of pulmonary nodules in uniportal thoracoscopy. J Thorac Cardiovasc Surg 152:535-544 (2016).

17. Wu W, Parmar C, Grossmann P et al. Exploratory study to identify radiomics classifiers for lung cancer histology. Front Oncol 6:71 (2016).

18. Kerr KM, Pelosi G, Austin JHM et al. Pleomorphic, spindle cell, and giant cell carcinoma; in Travis WD, Brambilla E, Burke AP, Marx A, Nicholson AG (eds): WHO Classification of Tumours of the Lung, Pleura, Thymus and Heart. Lyon, IARC, 88-90 (2015).

19. Soh, LK., Tsatsoulis, C. Texture analysis of SAR sea ice imagery using gray level co-occurrence matrices. IEEE Trans Geosci Remote Sens 37:780-795 (1999).

20. Zhang Y, Matuszewski BJ, Shark LK, Moore CJ. Medical image segmentation using new hybrid levelset method. In 2008 fifth international conference biomedical visualization: information visualization in medical and biomedical informatics, 71-76. IEEE (2008).

21. Son JY, Lee HY, Kim JH et al. Quantitative CT analysis of pulmonary ground-glass opacity nodules for distinguishing invasive adenocarcinoma from non-invasive or minimally invasive adenocarcinoma: the added value of using iodine mapping. Eur Radiol 26:43-54 (2016).

22. Mohanaiah, P., Sathyanarayana, P., \& GuruKumar, L. Image texture feature extraction using GLCM approach. International journal of scientific and research publications, 3(5), 1-5 (2013).

23. Ververidis D, Kotropoulos $C$. Fast and accurate sequential floating forward feature selection with the Bayes classifier applied to speech emotion recognition. Signal Process 88(12):2956-2970 (2008).

24. Jain AK, Duin RPW, Mao J. Statistical pattern recognition: A review. IEEE Transactions on pattern analysis and machine intelligence, 22(1), 4-37 (2000).

25. Cawley GC, Talbot NL. Efficient leave-one-out cross-validation of kernel fisher discriminant classifiers. Pattern Recognit 36:2585-2592 (2003).

26. MacMahon H, Naidich DP, Goo JM et al. Guidelines for Management of Incidental Pulmonary Nodules Detected on CT Images: From the Fleischner Society 2017. Radiology 284:228-243 (2017). 
27. Kazerooni EA, Armstrong MR, Amorosa JK et al. ACR CT Accreditation Program and the Lung Cancer Screening Program Designation. J Am Coll Radiol 13(2 Suppl):R30-R34 (2016).

28. Shaukat F, Raja G, Gooya A, Frangi AF. Fully automatic detection of lung nodules in CT images using a hybrid feature set. Med Phys 44:3615-3629 (2017).

29. Naqi SM, Sharif M, Yasmin M. Multistage segmentation model and SVM-ensemble for precise lung nodule detection. Int J Comput Assist Radiol Surg 13:1083-1095 (2018).

30. Shaukat F, Raja G, Ashraf R, Khalid S, Ahmad M, Ali A. Artificial neural network based classification of lung nodules in CT images using intensity, shape and texture features. J Ambient Intell Humaniz Comput 10:4135-4149 (2019).

31. Gu Y, Lu X, Zhang B et al. Automatic lung nodule detection using multi-scale dot noduleenhancement filter and weighted support vector machines in chest computed tomography. PLoS One 14(1):e0210551 (2019).

32. Saba T. Automated lung nodule detection and classification based on multiple classifiers voting. Microsc Res Tech 82:1601-1609 (2019).

33. Huidrom R, Chanu YJ, Singh KM. Pulmonary nodule detection on computed tomography using neuroevolutionary scheme. SIViP 13:53-60 (2019).

34. Jacobs $C$, van Rikxoort EM, Twellmann T et al. Automatic detection of subsolid pulmonary nodules in thoracic computed tomography images. Med Image Anal 18:374-384 (2014).

35. Khan SA, Nazir M, Khan MA et al. Lungs nodule detection framework from computed tomography images using support vector machine. Microsc Res Tech 82:1256-1266 (2019).

36. Naqi SM, Sharif M, Lali IU. A 3D nodule candidate detection method supported by hybrid features to reduce false positives in lung nodule detection. Multimedia Tools and Applications, 78(18), 2628726311 (2019).

\section{Figures}




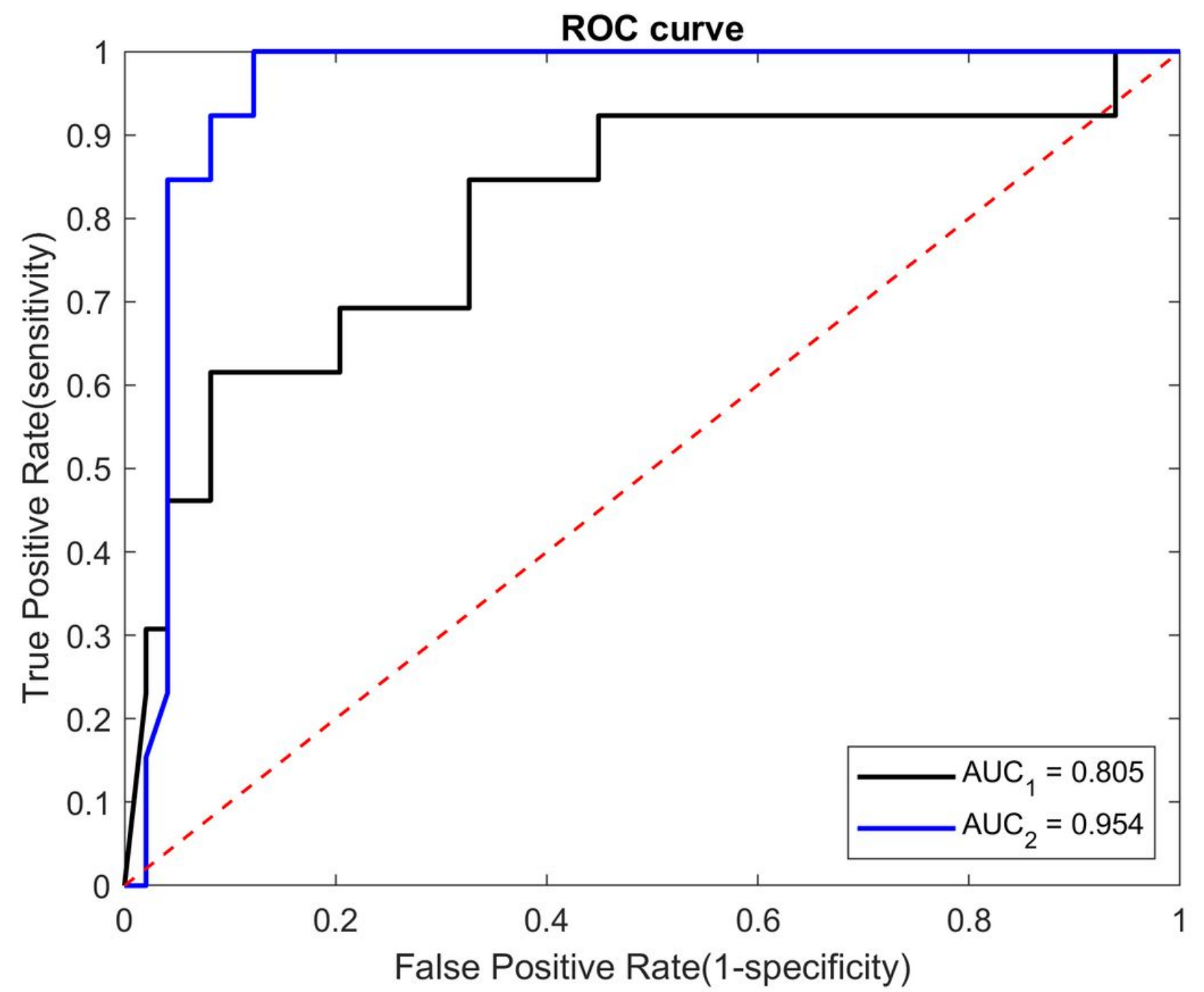

Figure 1

The Receiver operating characteristic (ROC) curve of the proposed solitary pulmonary capillary hemangioma/lepidic-predominant adenocarcinoma (SPCH/LPA) classification model (area under the curve $[A U C]=0.954)$ and baseline model $(A U C=0.805)$. 


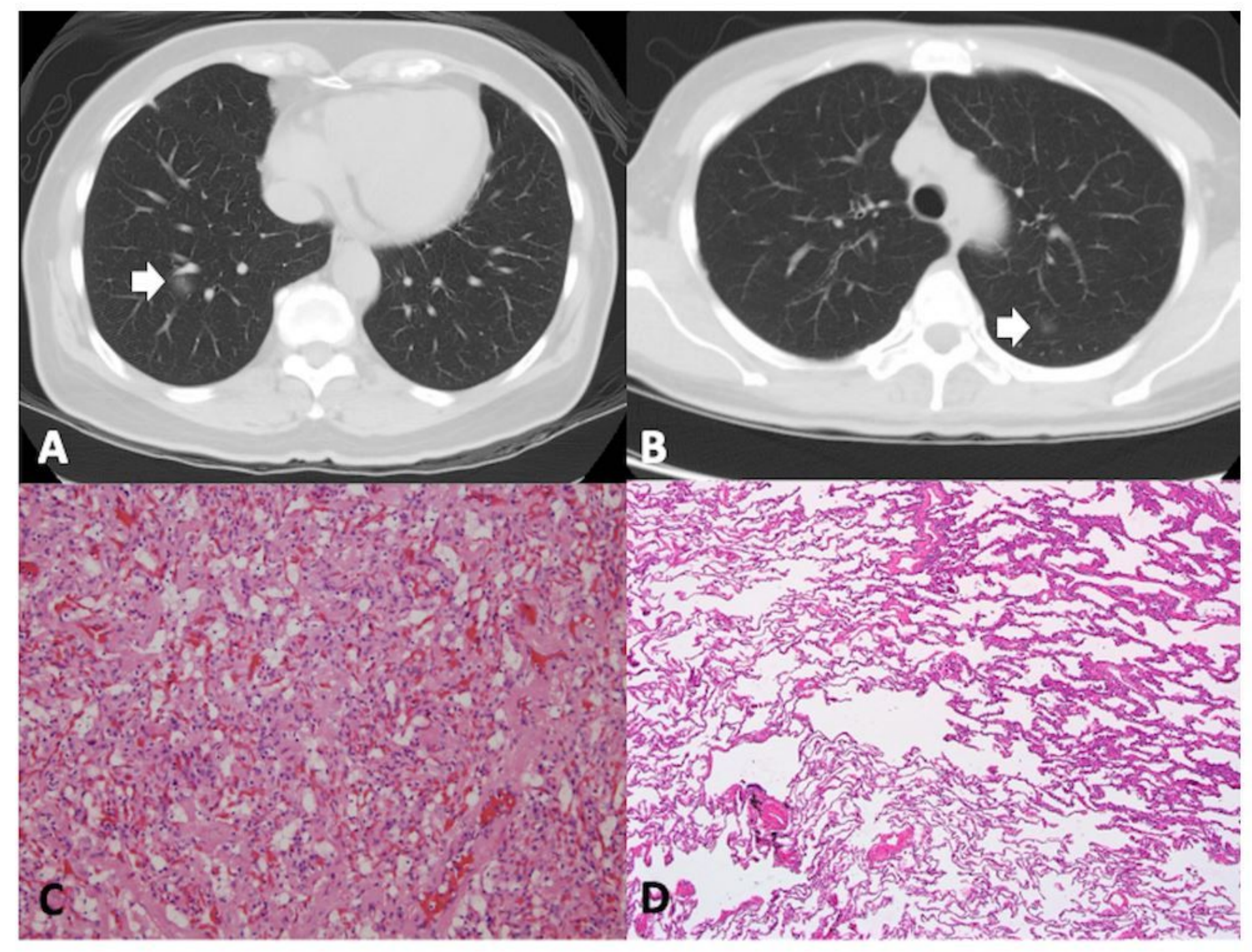

Figure 2

(A) Computed tomography (CT) image of solitary pulmonary capillary hemangioma (SPCH) usually presents as a pure ground-glass nodule, which is similar to the presentation of lepidic-predominant adenocarcinoma (LPA) of the lung. (B) CT image of LPA. (C) The thin-walled alveolar septa have being replaced by abundant capillary-sized vascular channels in the SPCH. (D) LPA consists of a cellular proliferation of pneumocytes along the surface of alveolar walls. 


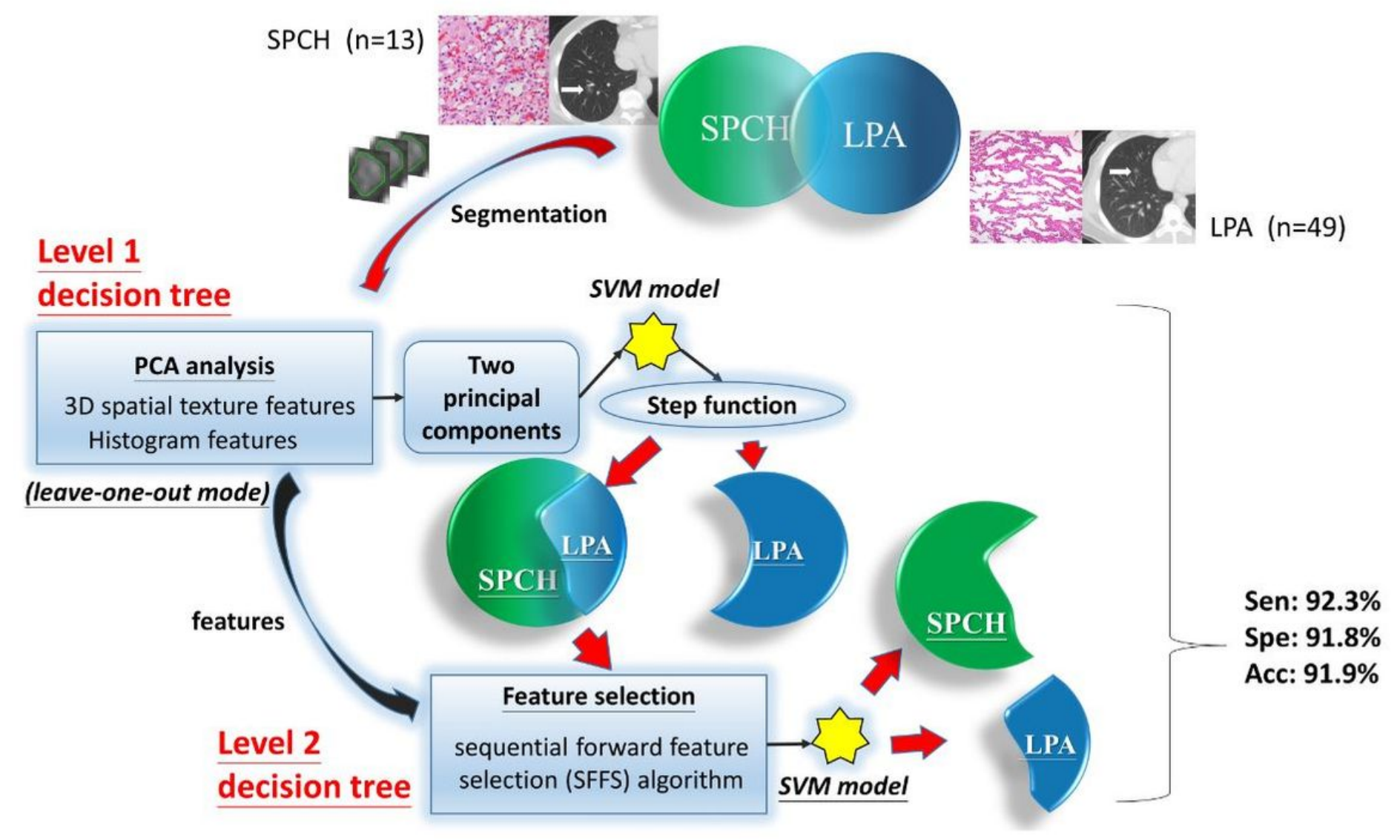

\section{Figure 3}

The schema of this study.

\section{Supplementary Files}

This is a list of supplementary files associated with this preprint. Click to download.

- SupplementaryTable12.docx 